\title{
Los datos y el azar en el currículo de educación básica y bachillerato en México: reflexiones desde la perspectiva internacional
}

\section{Data and randomness in the basic education and high school curricula in Mexico: insights from an international perspective}

DOI: https://doi.org/10.32870/dse.v0i22.717

Santiago Inzunza Cazares*

Eneyda Rocha Ruiz**

\begin{abstract}
Resumen
En el presente trabajo analizamos contenidos, aprendizajes esperados y orientaciones didácticas del currículo de estadística y probabilidad en la educación básica y bachillerato en México, con el propósito de reflexionar y relacionar con algunos currículos y recomendaciones de organismos que promueven la educación estadística a nivel internacional. Analizamos también las ideas fundamentales de la estadística y su presencia en el currículo mexicano. Los resultados muestran que el estudio de los datos y el azar está presente desde preescolar hasta bachillerato. La metodología de enseñanza en educación básica otorga importancia a contextos reales y al planteamiento de preguntas estadísticas para responder con los datos, pero en bachillerato se hace mayor énfasis en el cálculo estadístico. La tecnología para análisis de datos y simulación está prácticamente ausente en el currículo de todos los niveles.
\end{abstract}

Palabras clave: educación básica - educación media superior - estadística - enseñanza de las matemáticas - currículo de matemáticas.

\begin{abstract}
This paper analyzes contents, expected learning and didactic orientations of the curricula for statistics and probability in Mexico's elementary and high school education, with the aim of reflecting on and establishing relationships with some curricula and recommendations of international organizations that promote education on statistics. We also analyze the fundamental ideas of statistics and their presence in the Mexican curriculum. The results show that statistics and probability are present from preschool to high school. The methodology of teaching in basic education gives importance to real contexts and to asking statistical
\end{abstract}

* Doctor en Ciencias en la especialidad de Matemática Educativa. Líneas de investigación: didáctica de la estadística, uso de TIC en la educación matemática. Universidad Autónoma de Sinaloa. México. sinzunza@uas.edu.mx

** Doctora en educación. Línea de investigación: informática educativa. Universidad Autónoma de Sinaloa. México. eneyda@uas.edu.mx 
questions to be answered using the data, but in high school there is a greater emphasis on statistical procedures. The technology for data analysis and simulation is practically absent in the curriculum of all levels.

Key words: basic education - high school education - statistics - mathematics teaching -mathematics curriculum.

\section{Introducción}

En las últimas tres décadas, el estudio sobre los datos y el azar, considerado en las materias de estadística y probabilidad, ha tenido un crecimiento notable en el currículo de matemáticas de la educación básica y bachillerato (p.e., MEC, 2006; NCTM, 1989, 2000), debido a su importancia como herramienta metodológica, pero también por la relevancia que tiene el razonamiento, la alfabetización y el pensamiento estadístico en la sociedad moderna (Batanero et al., 2014), caracterizada por muchos estudiosos del tema como sociedad de la información y del conocimiento. Tal crecimiento se explica en gran medida por la revolución de los datos impulsada por el desarrollo de las Tecnologías de la Información y la Comunicación. Este hecho, a su vez, ha derivado en otro fenómeno conocido como cuantificación de la sociedad, generado por la necesidad de representar y resumir información en términos numéricos a través de gráficas, tablas estadísticas, porcentajes, promedios, correlaciones, modelos, variabilidad y otras medidas estadísticas, para describir e inferir el comportamiento de muestras, poblaciones o experimentos aleatorizados.

El proceso de inclusión de la estadística y la probabilidad en el currículo no ha sido uniforme en todos los países, tampoco ha estado exento de discusión y debate sobre los contenidos, su proporción en los programas de estudio y los enfoques de enseñanza (Watson, Chance, 2012). En este sentido, organismos e investigadores que promueven la educación estadística a nivel internacional, basándose en resultados proporcionados por la investigación, han emitido diversas recomendaciones sobre los fundamentos y las ideas centrales de la estadística y probabilidad que deben estar contenidas en los currículos de cada nivel educativo, así como las perspectivas de enseñanza más acordes a las necesidades de alfabetización y pensamiento estadístico de la sociedad actual. En algunos países se han retomado estas recomendaciones y las han plasmado en el currículo en los años recientes (por ejemplo, Nueva Zelanda, Inglaterra, Alemania y Estados Unidos), al grado que la estadística y probabilidad ocupan el mismo nivel de importancia que las áreas tradicionales de las matemáticas como aritmética, álgebra, geometría, trigonometría y cálculo.

En este contexto, es pertinente analizar el estatus y significado que tienen la estadística y la probabilidad en los niveles educativos preuniversitarios en México. Interesa analizar contenidos, aprendizajes esperados y orientaciones didácticas definidas en el currículo en los diferentes niveles educativos, con el propósito de caracterizar su enseñanza y hacer un contraste con

Diólo@os sobre Educación año 12 | número 23 | julio-diciembre 2021 | ISSN 2007-2171 
las recomendaciones y la tendencia del currículo internacional. Ello nos permitirá determinar similitudes y diferencias, e identificar posibles aspectos de mejora del currículo.

\section{La educación básica y el bachillerato en México}

La educación básica en México comprende tres niveles: preescolar, primaria y secundaria, mientras que la educación media superior comprende el bachillerato y la educación profesional técnica. Los niveles, grados y edad de los estudiantes para cursar cada uno de ellos se describen en la tabla 1.

Tabla 1. Número de años y edad de los estudiantes mexicanos por nivel escolar

\begin{tabular}{|l|c|c|}
\hline \multicolumn{1}{|c|}{ Nivel } & Grados & $\begin{array}{c}\text { Edad de los } \\
\text { estudiantes }\end{array}$ \\
\hline Preescolar & 3 & 3 a 5 años \\
\hline Primaria & 6 & 6 a 12 años \\
\hline Secundaria & 3 & 13 a 15 años \\
\hline Medio Superior & 3 & 16 a 18 años \\
\hline
\end{tabular}

En la educación básica (preescolar, primaria y secundaria), la Secretaría de Educación Pública (SEP) define programas de estudio únicos y con alcance nacional, no así en el nivel medio superior, donde la educación es impartida por diversos sistemas (federal, estatal y autónomo), cada uno con su propio programa de estudios. Sin embargo, en 2008, en el contexto de la Reforma Integral a la Educación Media Superior, la SEP elaboró un Marco Curricular Común que sirve de referente para articular las diferentes opciones educativas y definir un mismo perfil de egreso, a partir de un conjunto de competencias a desarrollar en el aula por parte de los estudiantes.

\section{El currículo de estadística y probabilidad en los niveles preuniversitarios}

Los temas de estadística y probabilidad han ocupado cada vez más espacio en el currículo escolar de los niveles preuniversitarios. Holmes (2003) señala que en Inglaterra, desde 1961 se introdujeron contenidos relacionados con datos y azar en cursos de bachillerato. Estos contenidos fueron extendidos a la escuela primaria y secundaria inglesa. Por su parte, en las décadas de 1980 y 1990 se publica en Estados Unidos el Curriculum and Evaluation Standards for School Mathematics (NCTM, 1989) y Principles and Standards for School Mathematics (NCTM, 2000), documentos curriculares en los que el eje de Análisis de Datos y Probabilidad aparece como parte integral del currículum de matemáticas (Scheaffer, Jacobbe, 2014).

En este mismo país, con el aval de la Sociedad Americana de Estadística, en 2005 se publica el documento "Guidelines for Assessment and Instruction in Statistics Education" (GAISE) (Franklin et al., 2005), el cual representa un marco para la educación estadística desde preesco- 
lar hasta bachillerato, con una estructura conceptual orientada al desarrollo de la alfabetización y el pensamiento estadístico de los estudiantes. En esta misma dirección, se publican los Common Core State Standards in Mathematics (CSSO, 2010), un referente fundamental para la enseñanza de las matemáticas desde preescolar hasta bachillerato en Estados Unidos, en los cuales la estadística y la probabildad ocupan un lugar importante desde el nivel preescolar.

Mientras tanto, en 1992, en Nueva Zelanda se publica el documento Mathematics in the New Zealand Curriculum (Ministery of Education, 2007), en el cual se incluye por primera vez la estadística y la probabilidad como área de enseñanza en todos los niveles educativos. En 2007 el eje curricular de matemáticas se renombra como Matemáticas y estadística, con lo cual se resalta la importancia que se otorga a esta última como área de estudio. Se incluyen tres grandes líneas de contenido estadístico en los ocho niveles de la educación preuniversitaria: investigación estadística, cultura estadística y probabilidad. Asimismo, en España la estadística tiene presencia en el currículo de matemáticas desde la escuela primaria, y se profundiza en la escuela secundaria y el bachillerato desde las última década del siglo pasado (Batanero, Artega, Gea, 2012).

En la región de Latinoamérica, y específicamente en México, en el Plan de Estudios de 1972 ya se incluyen temas de probabilidad y estadística a partir del $3^{\circ}$ grado; en el caso de los programas de estudio de educación secundaria incorporan este tipo de contenidos a partir de 1975, y en el caso de algunos programas del nivel medio superior desde finales de la década de 1970. El currículo del nivel medio superior consta de tres componentes formativos (básico, propedéutico y profesional). La estadística es parte del componente propedéutico, es decir, no forma parte del cuadro básico de materias, pero con base en su autonomía curricular, la mayoría de las instituciones educativas integran al menos un curso de estadística y probabilidad en su currículo, ya sea de manera optativa u obligatoria.

En Costa Rica, desde la década de 1990 se incorporan contenidos de estadística descriptiva en los planes de estudio de primaria y bachillerato. En Chile, a partir de 1998 aparecen contenidos de estadística en los programas de estudio de Educación Media, y en 2002 se incorpora el eje de Estadística y Probabilidad como un elemento de innovación curricular del grado 6 (finales de primaria) al grado 12 (último grado de bachillerato), en 2009 se amplían y profundizan los contenidos correspondientes a datos y azar, en los cuales aparecen de manera explícita temas de muestreo, inferencia estadística y elementos de razonamiento inferencial a partir del séptimo año.

En Brasil, desde 1997 se incluye la estadística y la probabilidad como parte del currículo de matemáticas de la educación básica (Campos, Carzola, Kataoka, 2011). En el caso de Colombia, Gómez (2016) señala que desde 1998 los lineamientos curriculares incluyen el eje de Pensamiento Aleatorio y Sistemas de Datos, mismo que se divide en tres ejes temáticos: organización de datos, medidas de posición y variabilidad, probabilidad e inferencia. Mientras tanto, Salcedo 
(2006) cita que en 1972 en Venezuela se introducen por vez primera tópicos de probabilidad y estadística en el currículo de matemáticas del bachillerato, los cuales se profundizan en la reforma de 1990, a la luz de recomendaciones internacionales.

\section{Marco conceptual}

\section{Ideas estadísticas fundamentales de estadística y probabilidad}

En la búsqueda por definir un conjunto de ideas fundamentales de estadística y probabilidad que deben ser aprendidas por los estudiantes antes de concluir el bachillerato, Burrill y Biehler (2011) proponen las siguientes:

1. Datos. Los estudiantes deben reconocer los diferentes tipos de datos y los distintos diseños de recolección; además, deben ser conscientes que un diseño de recolección inadecuado tiene efecto en los resultados y conclusiones de una investigación.

2. Variabilidad. La variabilidad es una característica intrínseca de los datos. Los estudiantes deben anticipar la variabilidad para plantear preguntas estadísticas en un problema, reconocer la variabilidad debida a los diseños de recolección de datos, dar cuenta de la variabilidad en el análisis por medio de distribuciones, e interpretarla generalizando más allá de los datos disponibles.

3. Distribución. Los datos deben ser vistos como colecciones de datos, los cuales pueden ser descritos por su tendencia central, variabilidad y forma. Los datos pueden provenir de mediciones de variables estadísticas (distribución de datos), de mediciones de variables aleatorias o generados por modelos (distribuciones aleatorias) u obtenidos como resúmenes de muestras, para dar lugar a distribuciones muestrales.

4. Representaciones. Las gráficas y tablas estadísticas son fundamentales en todas las etapas del análisis de datos, ayudan a la visualización de patrones y tendencias; constituyen una parte importante del trabajo estadístico y son la cara más visible de la estadística en los medios de comunicación.

5. Asociación y modelación entre dos variables. En estadística es muy frecuente el análisis de dos o más variables de manera simultánea, para determinar la asociacion entre ellas y proceder a la construcción de modelos predictivos. Los estudiantes deben estar preparados para responder preguntas que requieran explorar relaciones entre dos o más variables (pensamiento multivariable).

6. Modelos de probabilidad. El estudio de la probabilidad no puede ser desligado del estudio de la estadística; aunque hay diferentes visiones de la cantidad de probabilidad que debe ser enseñada en un curso de estadística, hay un acuerdo prácticamente generalizado de que el enfoque clásico por sí solo es insuficiente y debe ser complementado con el enfoque frecuencial y subjetivo de la probabilidad. 
7. Muestreo e inferencia. La enseñanza de la inferencia estadística se ha realizado predominantemente desde un enfoque formal, el cual requiere buenos antecedentes de teoría de probabilidad. En los años recientes ha surgido una línea de investigación conocida como inferencia estadística informal. La idea central de este enfoque radica en la simulación repetida del muestreo de una población o proceso para generar distribuciones muestrales de un estadístico de manera empírica.

\section{Lineamientos para la evaluación e instrucción en educación estadística}

El reporte GAISE (Franklin et al., 2005) propone un modelo central de enseñanza, basado en la resolución de problemas estadísticos como un proceso investigativo de cuatro componentes: formular preguntas para responder con los datos, recolectar los datos, analizar los datos e interpretar resultados para responder las preguntas planteadas. En este proceso, la variabilidad y el contexto ocupan un papel central, desde el planteamiento de preguntas hasta la interpretación de resultados. En el proceso de resolver problemas estadísticos se establecen tres niveles de maduración ( $A$, B y C) que dependen del desarrollo de la alfabetización estadística.

En cuanto al enfoque de enseñanza y el contenido de probabilidad en el currículo, el reporte GAISE visualiza la probabilidad como una herramienta para la estadística. En este sentido, se considera que debe explorarse la relación entre enfoque clásico y frecuencial para comparar probabilidades teóricas y frecuencias observadas como una forma de establecer relación entre modelo y realidad. Usar la probabilidad para comprender la aleatorización en el trabajo estadístico en el caso del muestreo y el diseño de experimentos.

\section{Método}

La investigación que hemos utilizado es de carácter documental (Bardin, 2006). Las fuentes de información fueron los documentos curriculares oficiales más actuales. Se analizaron los contenidos, aprendizajes esperados y orientaciones didácticas descritas en los documentos, para contrastarlos con lo propuesto por Burrill y Biehler (2011) y Franklin et al. (2005). En cada documento curricular se revisó con detalle la presencia de las ideas fundamentales descritas por Burrill y Biehler (2011); asimismo, se analizaron en el texto, en enunciados y declaraciones que informan sobre los contenidos, aprendizajes esperados y orientaciones didácticas.

\section{Resultados y discusión}

El estudio de la estadística aparece desde el nivel preescolar, mientras que el estudio de la probabilidad inicia en quinto grado de primaria en el currículo mexicano. En la educación básica, ambos contenidos se incluyen como temas en la materia de matemáticas, mientras que en el nivel medio superior los contenidos son parte de una materia por sí sola, denominada por lo general Probabilidad y Estadística, o simplemente Estadística. 
Tabla 2. Contenidos de estadística y probabilidad en la educación básica y bachillerato en México

\begin{tabular}{|c|c|c|c|c|}
\hline Tema & Preescolar & Primaria & Secundaria & Bachillerato \\
\hline $\begin{array}{l}\text { Recolección de } \\
\text { los datos }\end{array}$ & $\begin{array}{l}\text { Observación, } \\
\text { encuestas, } \\
\text { entrevista, } \\
\text { consulta de } \\
\text { información. }\end{array}$ & $\begin{array}{l}\text { Encuestas, ob- } \\
\text { servación, entre- } \\
\text { vista, consulta de } \\
\text { información. }\end{array}$ & $\begin{array}{l}\text { Encuestas, obser- } \\
\text { vación, entrevista, } \\
\text { consulta de infor- } \\
\text { mación. }\end{array}$ & $\begin{array}{l}\text { Encuesta, entrevista, obser- } \\
\text { vación, experimentación. } \\
\text { Muestreo probabilístico y no } \\
\text { probabilístico. }\end{array}$ \\
\hline $\begin{array}{l}\text { Organización, } \\
\text { representa- } \\
\text { ción, interpre- } \\
\text { tación de datos }\end{array}$ & $\begin{array}{l}\text { Pictogramas, } \\
\text { tablas. }\end{array}$ & $\begin{array}{l}\text { Pictogramas, } \\
\text { tablas, diagra- } \\
\text { mas de barras y } \\
\text { circulares. }\end{array}$ & $\begin{array}{l}\text { Gráficas circulares, } \\
\text { histogramas, polígo- } \\
\text { nos de frecuencia y } \\
\text { gráficas de línea. }\end{array}$ & $\begin{array}{l}\text { Distribuciones de frecuencia. } \\
\text { Gráficas circulares, histogramas, } \\
\text { polígonos de frecuencia y ojivas. }\end{array}$ \\
\hline $\begin{array}{l}\text { Medidas des- } \\
\text { criptivas de los } \\
\text { datos }\end{array}$ & & $\begin{array}{l}\text { Moda, media } \\
\text { aritmética y ran- } \\
\text { go de un conjun- } \\
\text { to de datos. }\end{array}$ & $\begin{array}{l}\text { Medidas de ten- } \\
\text { dencia central } \\
\text { (moda, media } \\
\text { aritmética y me- } \\
\text { diana) y rango y } \\
\text { desviación media } \\
\text { de un conjunto de } \\
\text { datos. }\end{array}$ & $\begin{array}{l}\text { Medidas de tendencia central } \\
\text { (moda, media aritmética y } \\
\text { mediana) y medidas de dis- } \\
\text { persión (rango y desviación } \\
\text { media, varianza y desviación } \\
\text { estándar) de un conjunto de } \\
\text { datos. } \\
\text { Cuartiles, deciles y percenti- } \\
\text { les. } \\
\text { Correlación de dos variables y } \\
\text { regresión lineal. }\end{array}$ \\
\hline Probabilidad & & $\begin{array}{l}\text { Experimentos } \\
\text { aleatorios, regis- } \\
\text { tro de frecuen- } \\
\text { cias y espacio } \\
\text { muestral. }\end{array}$ & $\begin{array}{l}\text { Experimentos } \\
\text { aleatorios, proba- } \\
\text { bilidad frecuencial, } \\
\text { probabilidad teó- } \\
\text { rica, probabilidad } \\
\text { de eventos mutua- } \\
\text { mente excluyen- } \\
\text { tes. }\end{array}$ & $\begin{array}{l}\text { Enfoques de probabilidad, } \\
\text { eventos y espacio muestral. } \\
\text { Conjuntos y operaciones con } \\
\text { conjuntos. } \\
\text { Técnicas de conteo, diagrama } \\
\text { de árbol, permutaciones y } \\
\text { combinaciones. } \\
\text { Eventos dependientes e inde- } \\
\text { pendientes. } \\
\text { Distribuciones de probabilidad } \\
\text { binomial, normal, Poisson, Ji } \\
\text { Cuadrada. } \\
\text { Probabilidad condicional y } \\
\text { teorema de Bayes. }\end{array}$ \\
\hline
\end{tabular}


Los aprendizajes esperados en preescolar giran en torno a contestar preguntas en las que se necesita recabar datos, organizarlos en tablas y representarlos en pictogramas sencillos; de tal forma, en las orientaciones didácticas se propone un ciclo que parte de recopilar datos por medio de observaciones, encuestas y consulta de información, representar los datos con códigos personales o convencionales, organizar y registrar datos en una tabla o pictograma e interpretar los datos para responder las preguntas. Se sugiere el uso de material concreto para, posteriormente, pasarlo a un registro gráfico. Ejemplo de preguntas que se plantean: ¿cuántos hermanos tienen los integrantes del grupo?, ¿cuál de tres cuentos es el favorito del grupo?

Esta metodología de planteamiento de preguntas para responder con los datos está en el centro de diversas recomendaciones (Franklin et al., 2005; NCTM, 2000) y algunos currículos (por ejemplo: Nueva Zelanda y Estados Unidos), y representa el inicio del ciclo de investigación para resolver problemas estadísticos.

En los grados inferiores de la educación primaria (de $1^{\circ}$ a 3er grado) se continúa y profundiza los contenidos y aprendizajes esperados de preescolar. En estos grados se señala la importancia de que los alumnos aprendan a distinguir preguntas particulares referidas a un elemento (por ejemplo ¿qué come ese pájaro que estoy viendo?), de preguntas estadísticas referidas a un grupo o colectivo (por ejemplo, ¿qué comen los pájaros?). En el análisis de datos, las preguntas de interés son estas últimas (SEP, 2019). Estas orientaciones didácticas están en concordancia con las recomendaciones del reporte GAISE para el nivel primaria. En los grados superiores (de $4^{\circ}$ a $6^{\circ}$ grado) se amplía el uso de representaciones de datos a diagramas de barras y circulares. En los grados $5^{\circ}$ y $6^{\circ}$ se introducen las nociones de variables cualitativas y cuantitativas, y aparece por primera vez el cálculo de medidas descriptivas de centro y dispersión de los datos (moda, media aritmética y rango). En las orientaciones se sugiere la recolección de datos del contexto del salón de clases y la escuela, y el planteamiento de preguntas que requieren el uso de tales medidas descriptivas.

El estudio de la probabilidad inicia en $5^{\circ}$ año de primaria, se empieza introduciendo la distinción entre experiencia aleatoria y no aleatoria, la idea de espacio muestral y la determinación de sus elementos mediante el uso de diagramas de árbol. Los aprendizajes esperados consisten en identificar juegos en los que interviene el azar, y realizar experimentos para registrar los resultados en tablas de frecuencias relativas y absolutas (enfoque frecuencial de la probabilidad).

Mientras tanto, en secundaria los contenidos de estadística y probabilidad aparecen de manera continua en los tres grados. Los aprendizajes esperados en estadística enfatizan de nuevo la recolección y registros de datos, y un repertorio más amplio de representaciones para la organización e interpretación de los datos; además de los diagramas de barras y circulares, se incluyen los histogramas, polígonos de frecuencias y gráficas de línea (series de tiempo). En el cálculo de medidas descriptivas de los datos se consideran la media, mediana, moda, rango y la desviación media. Se orienta a la discusión sobre la medida de tendencia central más apropia- 
da para un tipo particular de datos y a utilizar estas medidas para hacer comparaciones entre conjuntos de datos.

Las orientaciones didácticas hacen hincapié en recopilar datos de contextos escolares como el salón de clases o asuntos de interés para los estudiantes que aparecen en los medios. Se proponen diferentes significados de la media: como reparto equitativo, como mejor estimación de la medida de un objeto medido varias veces y como número alrededor del cual se acumulan los datos. Se estudian las propiedades de la media y la mediana, y se resalta el hecho de que la mediana puede ser mejor medida que la media en algunas situaciones. Se orienta a los docentes a construir las gráficas manualmente para que los estudiantes comprendan cómo se construyen. En cambio, el uso de hoja de cálculo para construir histogramas y polígonos de frecuencia se sugiere de manera aislada y superficial.

En cuanto a probabilidad, se espera que los estudiantes realicen experimentos aleatorios y registren resultados como un acercamiento a la probabilidad frecuencial; se propone, además, que determinen la probabilidad teórica de un experimento aleatorio y de dos eventos mutuamente excluyentes, utilizando las reglas de la suma y de la multiplicación de probabilidades. Se proponen juegos de azar en las orientaciones didácticas y experimentos donde se contrasten ambas. En $2^{\circ}$ grado se propone el uso de simulación de fenómenos aleatorios mediante algún software solo como una recomendación, lo cual está en plena concordancia con las recomendaciones de diversos autores y el currículo internacional (Burrill, Biehler, 2011; Chaput, Girard, Henry, 2011).

En cuanto al nivel medio superior, no existen programas únicos a nivel nacional; sin embargo, a partir de 2008 hay una propuesta de currículo oficial por parte de la SEP, que tomaremos como referencia para el análisis. El curso de Estadística y Probabilidad I ofrece un repaso y profundización de los contenidos de la escuela secundaria: elementos básicos de algunas técnicas de muestreo como la encuesta, la entrevista, la observación y experimentación, se introducen los conceptos de población y muestra; representaciones gráficas (histograma, polígono de frecuencias, circular y acumulados) y distribuciones de frecuencias; medidas de tendencia central (media, mediana, moda) y de dispersión (rango, desviación media); se agregan la varianza y la desviación estándar, cuartiles, deciles y percentiles. La novedad en este nivel es la introducción al análisis de datos de dos variables (datos bivariados), en el que se incluyen los diagramas de dispersión, el cálculo de la correlación y la regresión lineal simple. Este tema es muy recomendado en el proyecto GAISE y es una de las ideas fundamentales de estadística para el bachillerato (Burrill y Biehler, 2011) debido a su importancia para desarrollar el pensamiento multivariado de los estudiantes e introducirlos a la teoría de la predicción. En algunos países como Costa Rica y Chile, aún no aparece esta temática en el currículo.

En el curso de Estadística y probabilidad II, aparecen los temas de conjuntos, técnicas de conteo y eventos mutuamente excluyentes, previo al cálculo de la probabilidad teórica y fre- 
cuencial. Distribuciones de probabilidad Bernoulli binomial, normal y Poisson, probabilidad condicional y teorema de Bayes. La inferencia estadística no forma parte del currículo oficial definido por la SEP, solo es considerada en el sistema de bachillerato CCH-UNAM, de manera opcional en el último semestre. El currículo de este nivel promueve el uso de cuatro temas transversales en diversos ejes (social, ambiental, salud y habilidades lectoras), como una forma de aplicar la estadística para resolver situaciones de la vida real y el entorno de los estudiantes.

En cuanto al uso de tecnología en la enseñanza de la estadística y la probabilidad en todos los niveles, apenas hay referencias superficiales en el currículo mexicano. Se sugiere su uso para la simulación de fenómenos aleatorios como un medio para estimar probabilidades teóricas, pero no hay referencias de su uso en el análisis y exploración de los datos, situación que es ampliamente sugerida en las recomendaciones internacionales y currículos de Nueva Zelanda, España y otros países.

Se observa que las ideas fundamentales definidas por Burrill y Biehler (2011) tienen presencia en algunos o en todos los niveles educativos. Por ejemplo, la idea de datos y sus técnicas de recolección es común en todos los niveles. La idea de distribución no aparece explícitamente, pero sí los conceptos que la integran, como las medidas de tendencia central y de variabilidad que, al igual que las representaciones gráficas de los datos, tienen una fuerte presencia en el currículo de estadística. La idea de probabilidad aparece al final de la escuela, primaria con un enfoque frecuencial, en secundaria y bachillerato se amplía su estudio con un enfoque más centrado en el uso de técnicas combinatorias que en frecuencias. La asociación y correlación tienen presencia hasta el bachillerato, sin embargo, la idea fundamental de inferencia estadística no forma parte del currículo en ninguno de los niveles, con excepción del sistema de bachillerato CCH-UNAM que la contempla en uno de dos cursos opcionales que ofrece en el último grado.

\section{Consideraciones finales}

En México, el estudio de los datos tiene presencia desde el nivel preescolar hasta el bachillerato, mientras que el estudio del azar inicia en el $5^{\circ}$ grado de la educación primaria, lo cual guarda similitud con el currículo internacional (países), reivindicando con ello que la estadística y la probabilidad tienen un estatus importante en la educación de los ciudadanos. Sin embargo, existen algunas diferencias en cuanto a los contenidos en algunos niveles, particularmente en el bachillerato, donde se observa la ausencia de la inferencia estadística en el currículo oficial, ampliamente recomendado en el currículo internacional. Esto parece ser una tendencia en los países latinoamericanos, a excepción, por ejemplo, de Chile.

Los contenidos temáticos de recolección de datos, organización y representación de datos, y medidas descriptivas de los datos, constituyen la columna vertebral del contenido estadístico a lo largo de toda la educación básica y el bachillerato. Las técnicas de recolección de los datos (encuestas, observación, entrevista, consulta de información) son comunes en todos los niveles, 
pero aumentan de complejidad de un nivel a otro, concluyendo en el bachillerato con una descripción general de los métodos de muestreo aleatorio y no aleatorio.

De la misma manera, las representaciones gráficas evolucionan desde los pictogramas sencillos en preescolar a diagrama de barras y circulares en la primaria, a histogramas, polígonos de frecuencia y gráficas de línea para visualizar datos cuantitativos. Sin embargo, se encuentran ausentes en el currículo gráficas de tallo y hoja, de caja y de puntos, que son comunes en currículos de otros países. Las medidas descriptivas inician con el cálculo de frecuencias en preescolar, cálculo de la moda en primaria, y el cálculo de la media, moda y desviación media en secundaria, para ampliar a la desviación estándar, varianza y la correlación en el bachillerato.

Por su parte, la probabilidad en la escuela primaria inicia con el estudio de nociones de azar a través de experimentos aleatorios sencillos y cálculo de frecuencias de los resultados. En la educación secundaria se introduce el enfoque clásico y se promueve su contraste con el enfoque frecuencial, un aspecto muy recomendado en el currículo internacional. Sin embargo, en el bachillerato, la liga entre ambos enfoques no es fomentada y se hace mayor énfasis en el enfoque clásico con uso de técnicas combinatorias.

En cuanto a la metodología de enseñanza de estadística y probabilidad, observamos una tendencia uniforme en la educación básica, que sugiere el uso de contextos reales y significativos para los estudiantes y el planteamiento de preguntas estadísticas que se deben responder con los datos, lo cual, a nuestro juicio, representa una tendencia innovadora y acorde con la perspectiva de desarrollo del pensamiento estadístico en los estudiantes. Esta tendencia nos parece interrumpida en el bachillerato, que centra más su atención en etapas posteriores del ciclo de investigación estadística, como es el análisis de los datos.

El uso de la tecnología computacional en el análisis de datos y la simulación de fenómenos aleatorios solo tiene referencias superficiales en todos los niveles educativos, lo que constituye la mayor diferencia con el currículo internacional. Sin duda, esta es una asignatura pendiente, al igual que la ausencia de la inferencia estadística en el bachillerato, que debe mejorarse en el currículo mexicano.

\section{Referencias}

Bardin, L. (2002). Análisis de contenido. Madrid: Ediciones Akal.

Batanero, C.; P. Arteaga; M. M. Gea (2012). El currículo de estadística: reflexiones desde una perspectiva internacional. Revista UNO, 59, 9-17.

Batanero, C.; M. M. Gea; P. Arteaga; J. M. Contreras (2014). La estadística en la educación obligatoria: análisis del currículo español. Revista Digital Matemática, Educación e Internet, 14(2), 1-14.

Burrill, G.; R. Biehler (2011). Fundamental Statistical Ideas in the School Curriculum and in Training Teachers. En C. Batanero; G. Burrill; C. Reading (eds.). Teaching Statistics in School 
Mathematics-Challenges for Teaching and Teacher Education: A Joint ICMI/IASE Study. Nueva York: Springer Science+Business Media, 57-69.

Campos, T.; I. Carzola; V. Kataoka (2011). Statistics School Curricula in Brazil. En C. Batanero; G. Burrill; C. Reading (eds.). Teaching Statistics in School Mathematics Challenges for Teaching and Teacher Education: A Joint ICMI/IASE Study. Nueva York: Springer Science+Business Media, 5-8.

CCSO (2010). Common Core State Standards in Mathematics. Council of Chief State School Officers. http://www.corestandards.org/Math/

Chaput, B.; J. Girard; M. Henry (2011). Frequentist Approach: Modelling and Simulation in Statistics and Probability Teaching. En Batanero, C.; G. Burrill; C. Reading (eds.). Teaching Statistics in School Mathematics Challenges for Teaching and Teacher Education: A Joint ICMI/IASE Study. Nueva York: Springer Science+Business Media, 85-96.

Forbes, S. (2014). The Coming of Age of Statistics Education in New Zealand, and its Influence Internationally. Journal of Statistics Education, 22(2), 1-19.

http://jse.amstat.org/v22n2/forbes.pdf

Franklin, C.; G. Kader; D. Mewborn; J. Moreno; R. Peck; M. Perry; R. Scheaffer (2005). Guidelines for Assessment and Instruction in Statistics Education (GAISE) Report. Alexandria: American Statistical Association.

https://www.amstat.org/asa/files/pdfs/GAISE/GAISEPreK-12 Full.pdf

Gómez, E. (2016). Estadística y probabilidad en el currículo colombiano para educación básica y media. Artículo presentado en el XXVI Simposio Internacional de Estadística, Sucre. Agosto (8-12).

https://docplayer.es/42683957-Estadistica-y-probabilidad-en-el-curriculo-colombiano-para-educacion-basica-y-media.html

Holmes, P. (2003). 50 Years of Statistics Teaching in English Schools: Some Milestones. Journal of the Royal Statistical Society. Series D (The Statistician), 52(4), 439-474.

MEC (2006). Real Decreto 1513/2006, de 7 de diciembre, por el que se establecen las enseñanzas mínimas de la Educación primaria. España: Ministerio de Educación, Cultura y Deporte. https://www.boe.es/buscar/pdf/2006/BOE-A-2006-21409-consolidado.pdf

National Council of Teachers of Mathematics (1989). Curriculum and Evaluation Standards for School Mathematics. Reston: NCTM.

National Council of Teachers of Mathematics (2000). Principles and Standards for School Mathematics. Reston: NCTM.

Ministery of Education (2007). Mathematics and Statistics Currículum. https://nzcurriculum.tki.org.nz/The-New-Zealand-Curriculum/Mathematics-and-statistics

Salcedo, A. (2006). Statistics Education in Venezuela: The Case of Elementary and Middle School. Proceedings of the Seven International Conference on Teaching Statistics, Salvador Bahía, 
Brazil. Voorburg: International Statistical Institute.

http://iase-web.org/documents/papers/icots7/C125.pdf?1402524966

Scheaffer, R.; T. Jacobbe (2014). Statistics Education in the K-12 Schools of the United States: a Brief History. Journal of Statistics Education, 22(2). http://jse.amstat.org/v22n2/scheaffer.pdf Secretaría de Educación Pública (2018). Programa de Estudio de Probabilidad y Estadística II. México: SEP.

https:/www.dgb.sep.gob.mx/informacion-academica/programas-de-estudio/CFP/6to-Semestre/Probabilidad-y-Estadistica-ll.pdf

Secretaría de Educación Pública (2019). Plan y Programas de Estudio para la Educación Básica. México: SEP. https://www.planyprogramasdestudio.sep.gob.mx

Watson, J.; B. Chance (2012). Building Intuitions about Statistical Inference Based on Resampling. Australian Senior Mathematics Journal, 26(1), 6-18. 